\title{
1 Evolutionary insights into Mariner-like elements in Apis 2 species
}

3 Kakeru Yokoi ${ }^{1} *$, Kiyoshi Kimura ${ }^{2}$, Hidemasa Bono ${ }^{3,4}$

41 Insect Genome Research and Engineering Unit, Division of Applied Genetics, Institute of

5 Agrobiological Sciences (NIAS), National Agriculture and Food Research Organization

6 (NARO), 1-2 Owashi, Tsukuba, Ibaraki 305-8634, Japan

72 Honeybee Research Group, Division of Animal Breeding and Reproduction, National Institute

8 of Livestock and Grassland Science (NILGS), National Agriculture and Food Research

9 Organization (NARO), Tsukuba, 2 Ikenodai, Tsukuba, Ibaraki 305-0901, Japan

103 Database Center for Life Science (DBCLS), Joint Support-Center for Data Science Research,

11 Research Organization of Information and Systems, 1111 Yata, Mishima, Shizuoka 411-8540,

12 Japan

134 Program of Biomedical Science, Graduate School of Integrated Sciences for Life, Hiroshima

14 University, 3-10-23 Kagamiyama, Higashi-Hiroshima City, Hiroshima, 739-0046, Japan

$16 *$ Corresponding author: Kakeru Yokoi

17 E-mail addresses:

18 Kakeru Yokoi: yokoi123@affrc.go.jp

19 Kiyoshi Kimura: kimura@affrc.go.jp

20 Hidemasa Bono: bonohu@hiroshima-u.ac.jp

\section{Abstract}

25 Background: Mariner and mariner-like elements (MLEs) are distributed in various species and

26 their sequences are highly diverse. In previous reports, a few transposable element in the

27 genomes of Apis species mainly consist of mariner and MLE. For further insight of Apis MLEs,

28 detailed classifications of Apis MLE and sequences analysis of long MLEs, which may

29 potentially encode the transposase, are needed. 
30 Results: Much more MLEs were detected in A. mellifera genome compared to other Apis

31 species genomes. They were classified into 31 Drosophila MLE classes. In this classification,

32 almost all of MLEs were classified into the three classes belonging to mellifera subfamilies,

33 suggesting that Apis MLEs which exist thorough Apis species derived from single MLE

34 belonging to mellifera subfamily. Phylogenetic analysis using MLEs in the three classes showed

35 that there two types of clusters, of which one consist of multiple Apis species MLEs, and others

36 of only A. mellifera MLEs. Long MLEs analysis showed that only one long MLE encoding

37 complete transposase was found in all Apis genome except for A. mellifera genome, and the

38 MLE and multiple MLEs similar to it were found in A. mellifera genome. The analysis also

39 showed that other several long MLEs encoding complete transposase were found only in $A$.

40 mellifera genome.

41 Conclusions: Almost all of Apis MLEs are mellifera subfamilies. Of these MLEs, one types of

42 them settled in Apis species and burst in A. mellifera genome. The other one of MLEs invaded

43 into A. mellifera genome by horizontal transfer and exploded in A. mellifera genome. This is the

44 first example of the finer aspects of MLE evolution among closely related species,

\section{$46 \quad$ Key words}

47 Apis mellifera, Apis cerana, Apis florea, Apis dorsata, Mariner-like-elements, transposase. 


\section{Background}

49 Mariner is a class II transposable element (TE) that moves within a host genome in a

50 "copy and paste style" manner [1-3]. Mariner was initially discovered in the Drosophila

51 mauritiana genome [4]. The length of first detected mariner (MOS1) is $1286 \mathrm{bp}$. TE similar to

52 Mariner were subsequently identified in the genomes of many plants, vertebrates, and

53 invertebrates, and named mariner-like elements (MLEs). The size of MLEs are about the same

54 as one of Mos1, but varies copy to copy. The copy number of MLEs varies from species to

55 species as well. For example, a copies of MLEs in the human genome is estimated about 14,000

56 [5] and one of in Apis mellifera is less than 2,000 copies (see below). Mariner and MLEs

57 encode a transposase that possesses a D,D34D motif, which is characteristic of mariner and

58 MLEs [3]. Another mariner-specific characteristic is the terminal inverted repeat (TIR) located

59 at both ends of mariner that contains transposase-binding sites.

60 MLE has been detected using PCR with specific primers designed form consensus

61 region of the transposase. Detected MLEs have been are classified their subfamilies (e.g.

62 irritans, mellifera, mauritana, cecropia, and capitata)[2,6,7]. Previous studies indicated that

63 mariner and MLE distributed in the genomes of many species via horizontal transfer [2].

64 Evolutionary, the invading element is thought to have exploded in the host's genome (burst) and

65 with the accumulation of mutations, the MLEs becomes immobilized gradually. Most of the

66 detected MLEs are thought to be non-autonomous.

67 The PCR-based methods cannot detect all mariner and MLEs in genomes because a MLE that

68 has lost the consensus region cannot be found in this way. These days, due to the availability of

69 whole genome sequence data and the lower cost of genome sequencing, the methods employed

70 to identify TE, including mariner and MLE, have been modified. One method involves the use

71 of "RepeatMasker" (Smit A.F.A., Hubley R. \& Green P. RepeatMasker Open 4.0. 2013-2015,

72 http://www.repeatmasker.org), which searches sequences in whole genome data that are similar

73 to consensus TE sequences registered in a sequence library (e.g. Repbase) [8]. This method

74 detects all types of TE and autonomous and non-autonomous elements in the whole genome

75 region, thereby tracking MLEs and providing further insights into the horizontal transfer of

76 MLEs between host species.

77 The genus Apis (honey bee species) consists of four major and several minor species,

78 and the following whole genome data of major 4 species are currently available (three Apis

79 species and three Apis cerana subspecies): A. mellifera (Am) [9], A. cerana japonica (Acj) [10],

80 A. cerana cerana Korea native (Ack) [11], A. cerana cerana China native (Acc) [12], A.

81 dorsata (Ad) [13], and A. florea (Af) (Table 1). Apis genomes contain small numbers of TE, 
82 which mainly consist of class II TE, particularly MLEs [10,14]. Thus, through analysis using

83 these whole genomes, and detected MLEs data, MLEs dynamics in Apis genomes could be

84 revealed. Being a small number of copies, in MLE evolutionary studies, Apis are one of the

85 suitable insects.

86 Difficulties have been associated with the classification of TE because of variations in

87 their sequences, as described above. Using whole genome information, six mariner consensus

88 sequences (Ammers 1-6) have been reported for A. mellifera [14]. However, this classification

89 is just the results of RepeatMasker therefore it is not well considered.

90 Recently, using Drosophila genome, Wallau et al., (2014) re-classified the MLEs in Drosophila

91 species into 36 classes (Dromars) [15]. This classification is more sophisticated logically correct.

92 The old PCR methods did not follow evolutionary back ground. In the present study, we

93 extracted Dromar sequences in each Dromar class and built the profile files of each Dromar

94 classes by Clustal Omega and hmmer programs with the Dromar sequences. Apis MLEs were

95 then classified using these profiles by nhmmer (DNA homology search with profile Hidden

96 Markov Models). Moreover, a phylogenetic analysis of the Apis MLE of each Dromar class was

97 performed (Fig. 1). The dynamics of long Apis MLEs, defined as larger than 1kbp in Apis

98 genomes, were revealed by using sequence analysis. Through these results, we provide novel

99 evolutionary insights into Apis MLEs.

100

101 Results

\section{MLEs in six Apis species}

103

104

105

106

107

108

109

110

111

112

113

RepeatMasker was used to detect MLEs in the Am, Ack, Acc, Acj, Af, and Ad genomes. The output files obtained are shown in Supporting data 1 and 2. Approximately 50 to 150 MLEs were detected in the Ack, Acc, Acj, Af, and Ad genomes, while 2147 MLEs were identified in the Am genome. Detailed and sequence data of the MLEs detected are shown in Supporting data 3 and 4, respectively. To establish whether the identification of many MLEs was $A$. mellifera species- or subspecies-specific, the detection of MLEs by RepeatMasker with the same conditions was performed with the A. mellifera subspecies, these are A. mellifera carnica (Amcar), A. mellifera caucasica (Amcau), A. mellifera intermissa (Ami), and A. mellifera mellifera (Amm) (Table 1). The RepeatMasker results of these A. mellifera were not markedly different from Am (Supporting data 1 and 2), and the numbers of MLEs in Amcar, Amcau, Ami and Amm were 2247, 2215, 1738 and 2237, respectively. The numbers of MLEs in these 
114 genomes were not markedly different from those in Am. Therefore, we decided to perform

115 further analyses against Am genome data. While processing the present study, new and high-

116 quality Acc genome data (Acc_new) were published [16]. To clarify whether the results of

117 RepeatMasker particularly in MLEs, were changed, the detection of MLEs by RepeatMasker

118 with the same conditions was performed. The results of RepeatMasker were not markedly

119 different between Acc and Acc_new (Supporting data 1 and 2), and the number of MLEs in Acc

120 and Acc_new were 147 and 200, respectively. Therefore, we decided to perform further

121 analyses of Acc genome data. Collectively, the results of MLE numbers in these Apis genomes

122 suggested that a small number of MLEs existed in Apis genomes, except for Am genome, and

123 some of these MLEs might burst within the Am genome in the process of evolution.

\section{Dromar profiling}

To build profiling data on 36 Dromar groups, we extracted Dromar sequences using 20

126 Drosophila genome sequences and the positional data of Dromar reported by Wallau et al.

127 (2014). We only obtained 1418 Dromar sequences from 3685 Dromar copies because some

128 Drosophila genome data were not available in the public database or the descriptions of some

129 scaffolds in Drosophila genome data differed from those in positional data (Supporting data 5).

130 Therefore, sequences for Dromar 3, 7, 18, 19, and 20 were not extracted. Consequently, the

131 nhmmer profiles of 31 Dromars for the classification of Apis MLEs were built from the

132 alignment results of each Dromar group (separated and merged alignment and nhmmer profiling

133 data are shown in Supporting data 6 and 7, respectively).

\section{Apis MLE classification}

135 Apis MLEs were classified using the extracted Apis MLE sequences and Dromar

136 profiles. The results of the classification are shown in Fig. 2, and detailed classification results

137 are provided in Supporting data 8. In total, 864 out of approximately 2000 Am MLEs were

138 classified into only mainly Dromar 14, Dromar 17 and Dromar 29 except for one into Dromar6,

139 which are all melliferra subfamilies, while no MLE were classified into the other subfamilies.

140 (Fig. 2). The MLEs of the other five species classified into mauritiana and mellifera subfamilies,

141 and most of MLEs were classified into Dromar 14, Dromar 17 and Dromar 29. A list of all

142 classified MLEs is shown in Supporting data 8. The results suggested that MLEs in Apis

143 genome mainly consist of melliferra subfamilies.

\section{Phylogenetic analysis of Apis MLE and Dromar sequences.}



belonging to each Dromar class (newick files for phylogenetic trees are shown in Supporting

147 data 9), and a phylogenetic tree of Dromar 14, Dromar 17, and Dromar 29 was constructed

148 based on alignment results because the majority of MLEs were classified into the three classes

149 (Fig. 3), and an original tree figure is shown in supporting data 10. In Dromar 14, there were

150 distinct clades in the phylogenetic tree (Fig. 3A). Two of these clades (clades I and III)

151 consisted of only Am MLEs, while the other clade included six Apis MLEs plus Dromars (clade

152 II). In Dromar 17, there were four distinct clades (Fig. 3B). One of them included only

153 Am_MLEs (clade I). Clades II and IV include six Apis MLEs while clade III included Dromars.

154 In the case of Dromar 29, there were five distinct clades (Fig. 3C). Clade I consisted of only

155 Am_MLEs while clade II included two Ad_MLEs and an Ad_MLE. Clade III included the six

156 Apis MLEs while clade IV consisted of Dromars plus one Am_MLE. These results suggested

157 that there are two groups of Apis MLEs. We hypothesized that some MLEs forming the

158 Am_MLE-only group in the phylogenetic trees may invade to A. mellifera genomes by

159 horizontal transfer and burst within the A. mellifera genome, while the other MLEs forming six

160 Apis MLEs settle in Apis genomes.

161

\section{Long MLE analysis}

163 MLEs extracted using RepeatMasker include collasped, non-functional and short

164 "junk" MLEs. Further elucidating the evolution insight of MLE in Apis, "long" MLE

165 were analyzed. The long MLEs are thought to maintain the potential mobile feature of

166 MLE. To confirm this hypothesis, we initially listed Apis MLEs larger than 1 kbp (long MLEs)

167 (Table 4 and Supporting data 11). The majority of long MLEs are Am_MLEs. Long Am_MLEs

168 were equally distributed in all chromosomes, except for the LG_8 chromosome (Fig. 4),

169 suggesting that the burst of long Am_MLEs is not a chromosomal-local but across these all

170 chromosomes.

171 To assess the dynamism of these long Apis MLEs within or among the six Apis

172 genomes, we obtained the predicted transposase amino acid sequences using TransDecorder

173 (Supporting data 12). A phylogenetic tree was constructed by using the transposase sequences

174 (Fig. 5). There were three clades. One included Am_, Acc_, Ack_and Acj_MLEs while the

175 other two included only Am_MLEs, suggesting that MLEs consisting of Am_, Acc_, Ack_, and

176 Acj_MLEs settled in Apis genomes, while those consisting of Am_MLEs invaded and burst in

177 A. mellifera genome. 

MLEs that code full-length transposases (sequence of more than 300 amino acids). We found Acc_MLE_100.p1, Acj_MLE_148.p1, and Ack_MLE_6.p1 in this clade and the three sequences were the same, except for one residue, with 99\% identity of "Apis cerana Mariner transposase" in NCBI-nr (Accession ID: BAB86288.1) (data not shown) [12]. To clarify whether the transposase also existed in Am, Ad and, Af genomes, we performed BLAST search (tblastn) against the Am, Ad and Af genomes. The tblastn results of Am showed that 73 MLEs with an alignment length $>300$ and e-value $<1 \mathrm{e}-100$ were hits (supplemental data 14) and the E_value of one of the 73 MLEs (sequence ID:NC_037644.1 range:12509588-12510547) was 0.0, which may have been a "settling MLE" from Apis species in the Am genome. The tblastn results of Ad and Af showed that a single sequence was a hit in each genome. (supplemental data 14). To investigate whether the Acc_MLE_10 settled in the Apis genomes, we compared the nucleotide sequences of the Acc_MLE_10 plus the flanking 500bp on both sides with the hit MLEs plus flanking 500bp on both sides in Am, Ack, Acj, Ad, Af (supporting data 15). More than $80 \%$ identities were observed in all combinations and the internal sites of the two sequences were mostly same while most outer sites in both sides were different, showing that the TIR of each MLE was conserved. Collectively, these results suggested that the MLEs settled through Apis genomes and burst in the Am genome.

We searched long MLEs in the two clades including only Am_MLEs that code fulllength transposases (sequence of more than 300 amino acids) (Fig. 5). Of the two clades, we found no transposase with a sequence of more than 300 amino acids and the longest transposase was Am_MLE_1186.p1 (271 aa) in the smaller clade. We performed a blastp search by using Am_MLE_1186.p1 as query against NCBI-nr. The top hit sequence was "Mariner Mos1 transposase [Stegodyphus mimosarum] (Accession ID: KFM57872.1), the percent identity, E-

202 value, and query cover of which were $51.16 \%$, 1e-96, and $94 \%$ respectively (data not shown).

203 Using Am_MLE_1186.p1, we performed a tblastn search against the six Apis genome with E-

204 value < 1e-100. We did not obtain hits in any genomes, except for the Am genome (Supporting 205 data 16). Among the hit sites in the Am genome, there were 5 sites plus one query itself that 206 encoded more than 250 aa. We found two full-length transposases, Am_MLE_182.p1 and 207 Am_MLE_284.p1, in the larger clade. Am_MLE_182.p1 was annotated as Camar1 transposase 208 (Accession ID: AAO12862.1) by blastp against NCBI-nr, the percent identity, E-value, and 209 query cover of which were $48.09 \%, 1 \mathrm{e}-120$, and $99 \%$ respectively (data not shown). In the case 210 of Am_MLE_182.p1, the same annotation results of Am_MLE_284.p1 were obtained.

211 Consequently, we performed a tblastn search against the six Apis genomes using the two 
212 Am_MLEs. Am_MLE_284.p1 hit one sequence with an alignment length > 300 and e-value <

213 1e-100 in each Af, Acj and Ack genome, but did not hit any sequence in the Acc and Ad

214 genomes, and the hit sites in the Af, Acj and Ack were the same as the Acc_MLE_100 tblastn

215 hit site as previously reported. However, the percent of identities of these hits were

216 approximately $45 \%$ (data not shown). In the Am genome, seven sequences were more than 300

217 amino acids in length and had 80\% identity (Supporting data 17). In the case of

218 Am_MLE_182.p1, there was no tblastn hit site against the Ack, Acj, Acc, Ad, or Af genome

219 (data not shown). In the Am genome, there were seven with a sequence length of more than 300

220 amino acids and $80 \%$ identity. The hit sites were same as the hits of Am_MLE_284.p1

221 (Supporting data 17). These results suggested that there were two types of MLEs,

222 Am_MLE_1186 and Am_MLE_284 types, which were only found in the Am genome and burst 223 within it.

\section{Discussion}

225 In the present study, we extracted MLEs from six Apis genomes. The number of

226 Am_MLEs was markedly higher than those of the other Apis species MLEs. MLEs were

227 classified into Dromar classes according to Hidden Markov Model (HMM) profiles made by

228 Dromar sequences [15]. Classification results revealed that the majority of Apis MLEs were

229 classified into mellifera subfamilies in old classification. The phylogenetic tree of each class

230 showed that these MLEs formed two types of clusters, of which one included only Am_MLEs,

231 while the other included the MLEs of six or multiple Apis species. Moreover, there was one

232 long MLE-encoding complete transposase, which might settle in the Ad, Af, Acc, Ack, and Acj

233 genomes and might explode in the Am genome, and two other types of long Am_MLEs that

234 only existed in the A. mellifera genome and might explode within it.

235 More Am MLEs were detected than the other five Apis MLEs. Differences in the MLE

236 numbers detected were not due to the reference genome qualities of the six Apis species. We

237 detected MLEs by RepeatMasker using multiple Apis genome data (Table 3). For example, new

238 Acc genome data were published during the processing of the present study [16], and we

239 performed MLE detection using these data. Although the contig N50 values of new Acc

240 genome data were more than 150-fold higher than that of the Acc genome used in the present

241 study, the numbers of MLEs detected were not markedly different between the two sets of

242 genome data. Moreover, we detected MLEs by multiple A. mellifera genomes, the contig N50

243 values of which varied. Approximately 1700 to 2200 MLEs were detected in these Am genomes.

244 Based on these results, differences in MLEs between not Am genome and Am genome were not 
245 due to artificial or technical differences, suggesting that different feature of the MLEs among

246 each Apis species seemed to due to evolutionary history of each species.

247 The initial study on the Am genome detected approximately 1100 MLEs in the genome,

248 and these MLEs were classified into six classes, AmMarl-6, which included members of the

249 mellifera, irritans, and rosa subfamilies [14]. In the present study, we detected approximately

2502000 MLEs from the latest version of the Am genome [9], and 864 MLEs of the detected MLEs

251 with E-value < 1e-5 were classified. All 864 MLEs were allocated to mellifera subfamilies. This

252 difference in classifications between the present and previous studies may be due to the

253 detection and classification method, nhmmer. nhmmer adopted a HMM model that detects

254 previously unrecognized sequence features [17]. In detail, nhmmer, hmmer for nucleotide, can

255 detect remote homologs as sensitively as possible [17]. Thus, we think nhmmer is suitable for

256 analyzing TEs, which are often mutated and highly varied. RepeatMakser also adopted nhmmer

257 and searched TEs with Repbase library. On the other hand, the Dromar classification is

258 considered to be sophisticated because the classification was achieved by multiple methods and

259 many genome data. We attempted to perform a new classification method for Apis MLEs that

260 combines nhmmer (HMM) and Dromar sequences, and provided detailed classifications and

261 novel insights into Apis MLEs. Therefore, our classification method using nhmmer and the

262 MLE sequences of the other species may be employed in a TE analysis. Based on our

263 classification in the present study, the majority of MLEs of Am, Acc, Ack, Acj, Ad, and Af

264 were classified into mellifera subfamilies, while a few were classified into mauritiana

265 subfamilies. The present results further supported Apis MLEs exploding in the Am genome.

266 Phylogenetic trees revealed that there were two types of clusters, one of which

267 consisted of only Am_MLEs, and the other of several Apis species MLEs. It is plausible that the

268 former type of MLE invaded the genome in horizontal transmission and exploded after the bees

269 diverged in speciation from other species of bees while MLEs of the latter type invaded the

270 genome in horizontal transmission and settled through Apis species genomes and explode in the

271 Am genome before the species divergence. This aspect was also consistent with the analysis of

272 the long MLEs, which have putative transposases sequence and may maintain mobility. In

273 addition, the long MLE that encoded the complete transposase, A. cerana Mariner transposase,

274 was not found in the Ad, Af, or Am genome. However, the MLE encoding the complete

275 transposase was identified in the Ad, Af, and Am genomes by a tblastn search because these

276 MLEs were detected as a length $<1 \mathrm{kbp}$ by RepeatMasker and were filtered out. One MLE that

277 encoded the same sequence of A. cerana Mariner transposase was found in the Am genome, and

278 several MLEs that encoded transposases that were highly similar to A. cerana Mariner 
279 transposase were also detected. These results suggested that the MLEs encoding A. cerana

280 Mariner transposase invade horizontally through Apis evolutions and "burst" in the Am genome,

281 but not in other Apis species. On the other hand, other types of Am_MLEs which carry the

282 transposase similar to Camar1 transposase [Chymomyza amoena] were only found in the Am

283 genome. These types of MLEs may invade the only Am genome by horizontal transfer and burst

284 within the Am genome. The reason why MLEs only burst in the Am genome remains unknown.

285 The origin of Apis honey bee is Asia, except for Am, whose origin is Europe-Africa. On the

286 other hand, in most beekeeping, the Am is used and is widespread in the all over world as

287 domestic insects. These facts may be related to this aspect. More detailed structural analysis of

288 Am and other Apis genome must be needed. This is the first example of the finer aspects of

289 MLE evolution among closely related species, and perhaps the first time in other TEs.

290 Integrating all the results, the evolutionary aspect of MLEs belonging to the Dromar is shown in 291 Figure 6.

292

\section{Conclusions}

We detected MLEs from the genome data of six Apis species, and performed nhmmer-

295 based Apis MLE classification and phylogenetic analyses. About over 50-100 times of

296 Am_MLEs were detected than these of the other Apis MLEs. Almost all of Apis MLEs

297 classified were classified into mellifera subfamilies. The long MLEs of which length are over

298 1kbp divided into two types. One type of MLEs settled in Apis genome and burst in Am

299 genome, the other invade into and burst in Am genome. We showed firstly provided evolutional

300 insight Apis MLEs (Fig. 6).

\section{Materials and Methods}

\section{MLE detection and extraction of sequence data}

The six Apis genome genomes plus other versions and several A. mellifera subspecies sequences used in the present study are shown in Table 1.

305 RepeatMasker (Smit A.F.A., Hubley R. \& Green P. RepeatMasker Open 4.0. 2013-

306 2015, http://www.repeatmasker.org) was used to detect TE in the six Apis genomes, as

307 described in our previous study [10]. RepeatMasker output (.tbl and .out) files are shown in

308 Supporting data 1 and 2, respectively. Data on MLE were extracted from outfiles, and a single 
309 ID was allocated to each detected element (Supporting data 3). To extract each mariner

310 sequence, positional data in Supporting data 3 and BLAST software were utilized (Supporting 311 data 4) [18].

\section{Classification of Apis MLEs}

313 To build the hmmer profiles of Dromars, Dromar sequences were extracted using the

314 Drosophila genome data and Dromar location data reported by Wallau et al. (2014) (Supporting

315 data 5) [15]. However, some Dromar sequences were not extracted because genome sequence

316 IDs in Drosophila genome data differed from those described in Dromar location data.

317 Therefore, several Dromars were not used in the present study. The sequences of each Dromar

318 were aligned by Clustal Omega (Clustal Omega - 1.2.4) [19], and the hmmer profiles of

319 Dromars were made by hmmer-building (HMMER 3.2.1) (Supporting data 6) [17]. Using the

320 nhmmer search program with merged Dromar hmm profiles (Supporting data 7), the Apis

321 MLEs similar to each Dromar profile were identified (E-values < 1e-5) [17]. The output data

322 and table results of nhmmer are shown in Supporting data 18 and 19 respectively. Based on

323 hmmer search results, each MLE was classified into hit Dromar. If a MLE was hit to multiple

324 Dromars, it was classified into Dromar with the lowest e-value.

\section{Phylogenetic analysis}

326 Dromars and classified MLE were aligned by Clustal Omega-1.2.4, an approximately-

327 maximum-likelihood phylogenetic analysis was performed using FastTree version 2.1.10

328 (original data are shown in Supporting data 8) [20], and phylogenetic trees were drawn in

329 MEGAX version 10.1.8-1 and iTOL version 5 [21,22].

\section{Long MLE analysis}

331 The distribution of long Am_MLEs in A. mellifera chromosomes was visualized by

332 ChromoMap [23]. To obtain the predicted transposase of long MLEs, TransDecorder version

333 5.5.0 was used with default settings (https://github.com/TransDecoder/TransDecoder/wiki).

334 EMBOSS needle (6.6.0.0) was used to compare the sequences of the long MLEs encoding the

335 complete transposase plus flanking site [24].

\section{Data availability}


337 Analyzed data and additional results have been uploaded to figshare as Supporting data.

338 These data are available via the DOI shown in the Supporting data legends.

\section{References}

340 1. Oosumi T, Belknap WR, Garlick B. Mariner transposons in humans. Nature. 1995;378:672-

341672.

342 2. Robertson HM. The Tcl-mariner superfamily of transposons in animals. Journal of insect

343 physiology. 1995;41:99-105.

344 3. Plasterk RH, Izsvák Z, Ivics Z. Resident aliens: the Tc1/mariner superfamily of transposable

345 elements. Trends in genetics. 1999;15:326-332.

346 4. Jacobson JW, Medhora MM, Hartl DL. Molecular structure of a somatically unstable

347 transposable element in Drosophila. Proceedings of the National Academy of Sciences.

$348 \quad 1986 ; 83: 8684-8688$.

349 5. Lander ES, Linton LM, Birren B, Nusbaum C, Zody MC, Baldwin J, et al. Initial sequencing

350 and analysis of the human genome. Nature 2001;409,860-921

351 6. Sumitani M, Lee JM, Hatakeyama M, Oishi K. Cloning and characterization of Acmar1, a

352 mariner-like element in the asiatic honey bee, Apis cerana japonica (Hymenoptera, Apocrita).

353 Archives of Insect Biochemistry and Physiology 2002;50:183-190.

354 7. Yamada K, Kawanishi Y, Yamada A, Tokuda G, Gurung RD, Sasaki T, et al. A novel cluster

355 of mariner-like elements belonging to mellifera subfamily from spiders and insects:

356 implications of recent horizontal transfer on the South-West Islands of Japan. Genetica.

$357 \quad 2014 ; 142: 149-160$.

358 8. Jurka J, Kapitonov VV, Pavlicek A, Klonowski P, Kohany O, Walichiewicz J. Repbase

359 Update, a database of eukaryotic repetitive elements. Cytogenetic and genome research.

$3602005 ; 110: 462-467$.

361 9. Wallberg A, Bunikis I, Pettersson OV, Mosbech M-B, Childers AK, Evans JD, et al. A

362 hybrid de novo genome assembly of the honeybee, Apis mellifera, with chromosome-length

363 scaffolds. BMC genomics. 2019;20:275.

364 10. Yokoi K, Uchiyama H, Wakamiya T, Yoshiyama M, Takahashi J-I, Nomura T, et al. The

365 draft genome sequence of the Japanese honey bee, Apis cerana japonica (Hymenoptera: Apidae).

366 European Journal of Entomology. 2018;115.

367 11. Park D, Jung JW, Choi B-S, Jayakodi M, Lee J, Lim J, et al. Uncovering the novel

368 characteristics of Asian honey bee, Apis cerana, by whole genome sequencing. BMC genomics.

$3692015 ; 16: 1$. 
370 12. Diao Q, Sun L, Zheng H, Zeng Z, Wang S, Xu S, et al. Genomic and transcriptomic analysis

371 of the Asian honeybee Apis cerana provides novel insights into honeybee biology. Scientific

372 Reports 2018;8:1-14.

373 13. Oppenheim S, Cao X, Rueppel O, Krongdang S, Phokasem P, DeSalle R, et al. Whole

374 Genome Sequencing and Assembly of the Asian Honey Bee Apis dorsata. Genome Biology and

375 Evolution. 2020;12:3677-3683.

376 14. Weinstock GM, Robinson GE, Gibbs RA, Weinstock GM, Weinstock GM, Robinson GE, et

377 al. Insights into social insects from the genome of the honeybee Apis mellifera. Nature

$378 \quad 2006 ; 443: 931-49$.

379 15. Wallau GL, Capy P, Loreto E, Hua-Van A. Genomic landscape and evolutionary dynamics

380 of mariner transposable elements within the Drosophila genus. BMC genomics. 2014;15:727.

381 16. Wang Z-L, Zhu Y-Q, Yan Q, Yan W-Y, Zheng H-J, Zeng Z-J. A Chromosome-Scale

382 Assembly of the Asian Honeybee Apis cerana Genome. Frontiers in Genetics. 2020;11:279.

383 17. Wheeler TJ, Eddy SR. nhmmer: DNA homology search with profile HMMs. Bioinformatics

$384 \quad 2013 ; 29: 2487-9$.

385 18. Altschul SF, Gish W, Miller W, Myers EW, Lipman DJ. Basic local alignment search tool.

386 Journal of molecular biology. 1990;215:403-410.

387 19. Sievers F, Wilm A, Dineen D, Gibson TJ, Karplus K, Li W, et al. Fast, scalable generation

388 of high-quality protein multiple sequence alignments using Clustal Omega. Molecular Systems

389 Biology. 2011;7:539.

390 20. Price MN, Dehal PS, Arkin AP. FastTree: Computing Large Minimum Evolution Trees with

391 Profiles instead of a Distance Matrix. Molecular biology and evolution. 2009 26:1641-50. A

392 21. Kumar S, Stecher G, Li M, Knyaz C, Tamura K. MEGA X: molecular evolutionary genetics

393 analysis across computing platforms. Molecular biology and evolution. 2018;35:1547-1549.

394 22. Letunic I, Bork P. Interactive Tree Of Life (iTOL) v4: recent updates and new developments.

395 Nucleic acids research. 2019;47:W256-W259.

396 23. Anand L. chromoMap: An R package for Interactive Visualization and Annotation of

397 Chromosomes. bioRxiv. 2019;605600.

398 24. Li W, Cowley A, Uludag M, Gur T, McWilliam H, Squizzato S, et al. The EMBL-EBI

399 bioinformatics web and programmatic tools framework. Nucleic acids research. 2015;43:W580-

400 W584.

\section{Declarations}

402 Ethics approval and consent to participate 
403 Not applicable

\section{Consent for publication}

405 Not applicable

406 Availability of data and materials

407 All supporting data have been uploaded in figshare (DOI:10.6084/m9.figshare.c.4894503)

408 Supporting data 1

409 Out files of RepeatMasker from six Apis genomes plus A. mellifera subspecies and new Apis

410 cerana cerana China native genome data. DOI:10.6084/m9.figshare.11914278

\section{Supporting data 2}

412 Tbl files of RepeatMasker from six Apis genomes plus A. mellifera subspecies and new Apis

413 cerana cerana China native genome data. DOI: 10.6084/m9.figshare.11984451

\section{Supporting data 3}

415 MLE data on six Apis species plus A. mellifera subspecies and new Apis cerana cerana China

416 native genome data. DOI: 10.6084/m9.figshare.11914263

\section{Supporting data 4}

418 MLE sequences. DOI: 10.6084/m9.figshare.11913309

\section{Supporting data 5}

420 Dromar sequences (Wallau et al., 2014) used in the present study.

421 DOI:10.6084/m9.figshare.11956350

\section{Supporting data 6}

423 Alignment and hmmer profile files of Dromars. DOI:10.6084/m9.figshare.11962614

\section{Supporting data 7}

425 Merged Dromar hmmer profile for MLE classifications. DOI:10.6084/m9.figshare.11953734

\section{Supporting data 8}


427 List of Apis MLEs and classification results DOI:10.6084/m9.figshare.12045189

\section{Supporting data 9}

429 Original data of the phylogenetic analysis in newick files. DOI:10.6084/m9.figshare.11955621

\section{Supporting data 10}

432 Original phylogenetic trees figures of Dromar14 (DOI:10.6084/m9.figshare.13235168),

433 Dromar17 (DOI:10.6084/m9.figshare.12798179) and Dromar 29

434 (DOI:10.6084/m9.figshare.12798263).

435

436 Supporting data 11

437 ID list of all long Apis MLEs. DOI: 10.6084/m9.figshare.13235168

438

439 Supporting data 12

440 Predicted amino acid sequence of long MLEs transposase. DOI: 10.6084/m9.figshare. 13237385

441

442 Supporting data 13

443 Original figures of phylogenetic trees for transposase of Apis long MLEs DOI:

$444 \quad 10.6084 / \mathrm{m} 9$. figshare.13237388

445

446 Supporting data 14

447 Tblastn results of Acc_MLE_100 transposase as query against Am genome with E-value < 1e-

448 100. DOI: 0.6084/m9.figshare. 13237397

449

450 Supporting data 15

451 The nucleotide sequences data of the Acc_MLE_10 plus flanking 500bp and long MLEs

452 encoding complete transposase plus flanking 500bp in Am, Ack, Acj, Ad, Af, and results of

453 compering of the Acc sequences with the other sequences.

454 DOI: 10.6084/m9.figshare. 13237427

455

456 Supporting data 16 
457 Tblastn results of Am_MLE_1186 against A. mellifera genome DOI:

$458 \quad 10.6084 / \mathrm{m} 9$. figshare. 13238258

459

460 Supporting data 17

461 Tblastn results of Am_MLE_182.p1 and Am_MLE_284 against A. mellifera genome. DOI:

462

463 Supporting data 18

464 Results of nhmmer of Apis MLEs against Dromar profiles. DOI:10.6084/m9.figshare.11953746

465

466 Supporting data 19

467 Result tables of nhmmer of Apis MLEs against Dromar profiles. DOI:

$468 \quad 10.6084 / \mathrm{m} 9$. figshare.11953752

469 Competing interests

470 The authors declare no conflict of interest. The funders had no role in the design of the study; in 471 the collection, analyses, or interpretation of data; in the writing of the manuscript, or in the

472 decision to publish the results.

\section{Funding}

474 This work was supported by ROIS-DS-JOINT (026RP2019 and 030RP2018) to KY and by the

475 National Bioscience Database Center of the Japan Science and Technology Agency (JST) and

476 Hiroshima Prefectural Government to HB.

\section{Authors' contributions}

478 Conceived and designed the experiments: K.Y., K.K., and H.B.

479 Analyzed the data: K.Y., K.K., and H.B.

480 Contributed to the writing of the manuscript: K.Y. and H.B.

481 All authors discussed the data and contributed to manuscript preparation. K.Y. supervised the

482 project.

483 All authors read and approved the final manuscript.

\section{Figures \& Tables}


bioRxiv preprint doi: https://doi.org/10.1101/2020.04.15.035063; this version posted November 30, 2020. The copyright holder for this preprint (which was not certified by peer review) is the author/funder, who has granted bioRxiv a license to display the preprint in perpetuity. It is made available under aCC-BY 4.0 International license.

485 Fig. 1 Scheme of data analyses in the present study

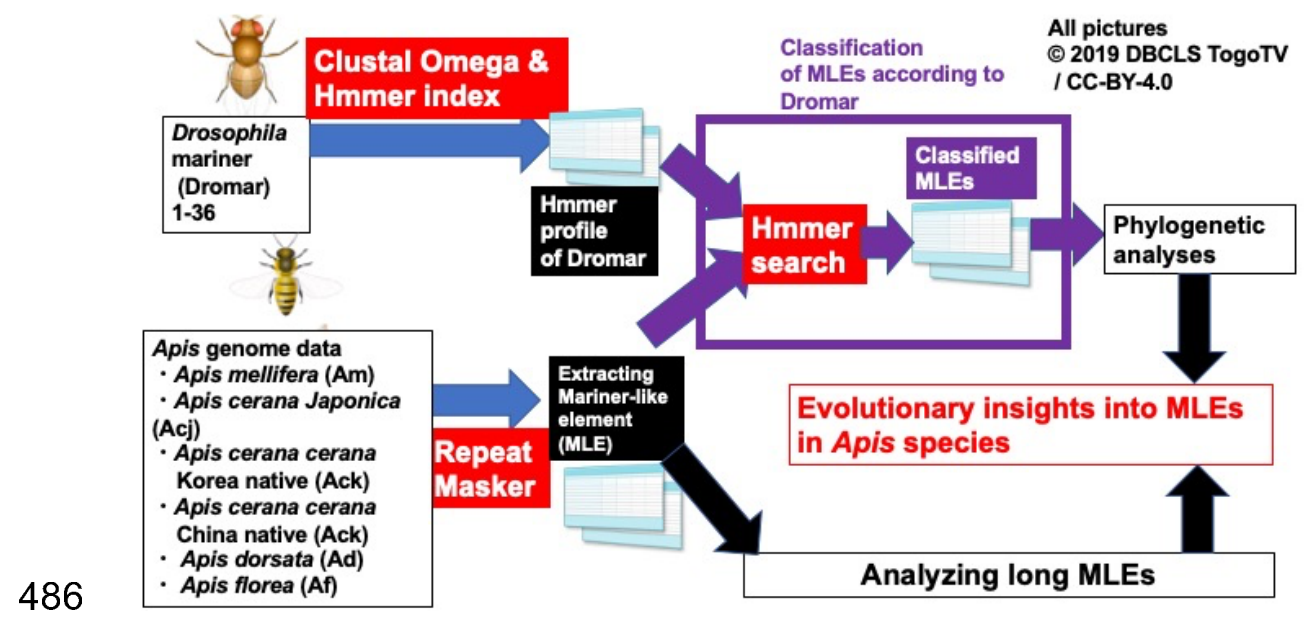


487 Fig. 2 Apis MLEs allocated to Dromars

488 The number of Apis MLEs allocated to each Dromar (e-value <1e-5). The left drawing shows 489 the molecular phylogeny of the genus Apis based on the study by Lo et al. (2010). The color of 490 each column indicates the subfamilies of mariner to which Dromar belongs, based on Wallau et 491 al. (2014). Unnumbered Dromars are those whose sequences could not be extracted from the 492 genome. The Dromars with white numbers filled in black comprised more than over 50 MLEs. 493 Asterisks indicate the Dromars consisting of MLEs from more than two Apis species. Detailed 494 classification lists are shown in Supporting data 8.

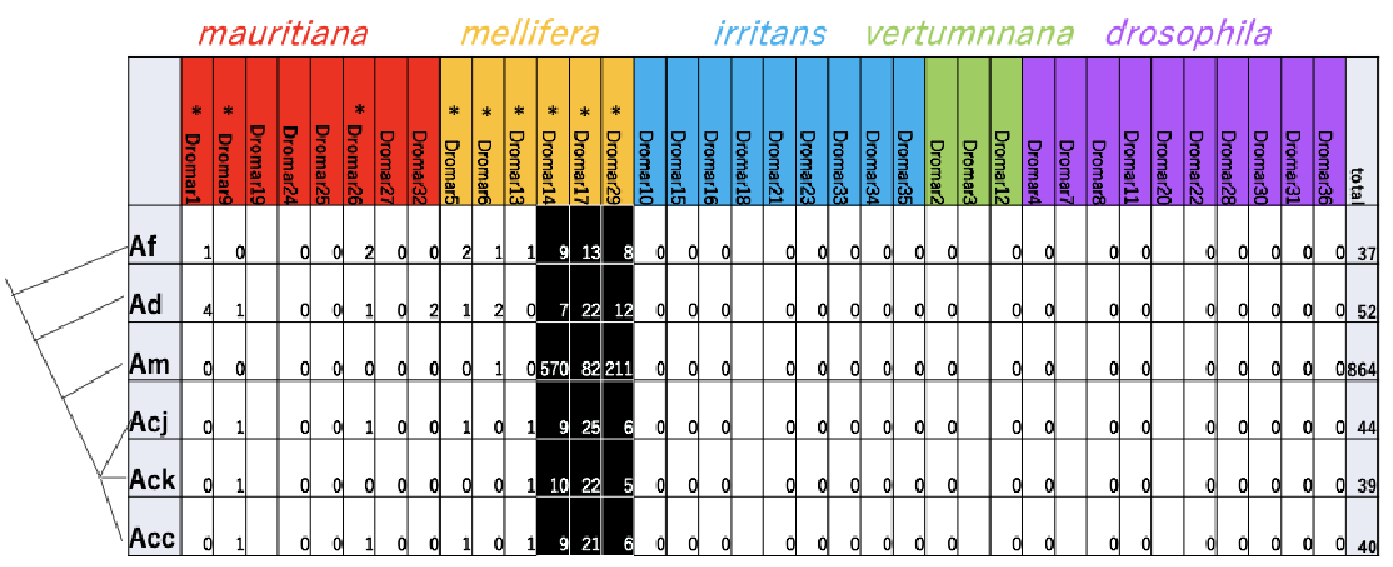


498 Fig. 3 Phylogenetic trees of three Dromar classes.

499 Phylogenetic trees based on the alignments of Apis MLEs of the three Dromar classes (Dromar

50014,17 , and 29), to which more than 50 MLEs are classified. Orange-, red-, and black-curved

501 lines in these figures indicate clusters including only Am_MLEs, multiple Apis species MLEs

502 and mainly Dromars. Roman numerals in the trees indicate independent clusters. Bootstrap

503 values symbolized the size of circles. The phylogenetic trees of other classes are shown in

504 supporting data 10. These original figures downloaded from iTOL are shown in supporting data

50510.

506 (A) Dromar 14

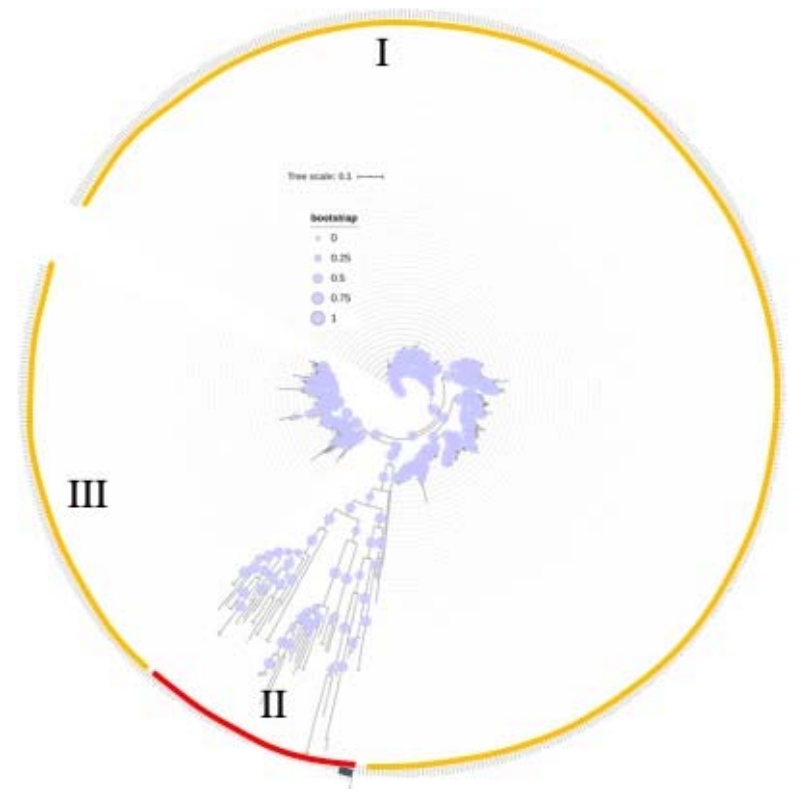

508 (B) Dromar 17 
bioRxiv preprint doi: https://doi.org/10.1101/2020.04.15.035063: this version posted November 30,2020 . The copyright holder for this preprint (which was not certified by peer review) is the author/funder, who has granted bioRxiv a license to display the preprint in perpetuity. It is made available under aCC-BY 4.0 International license.

510 (C) Dromar 29

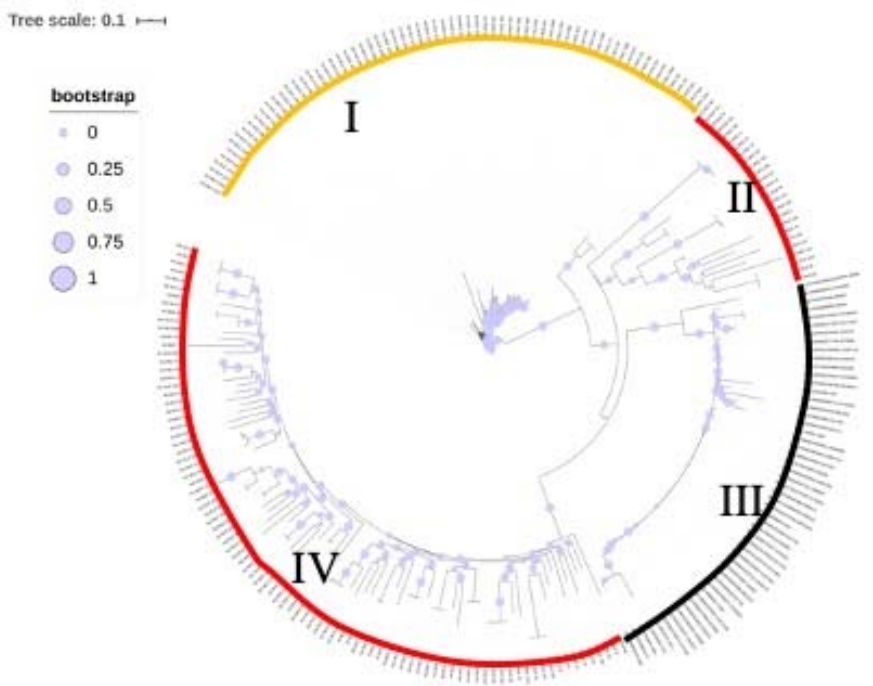

511

512

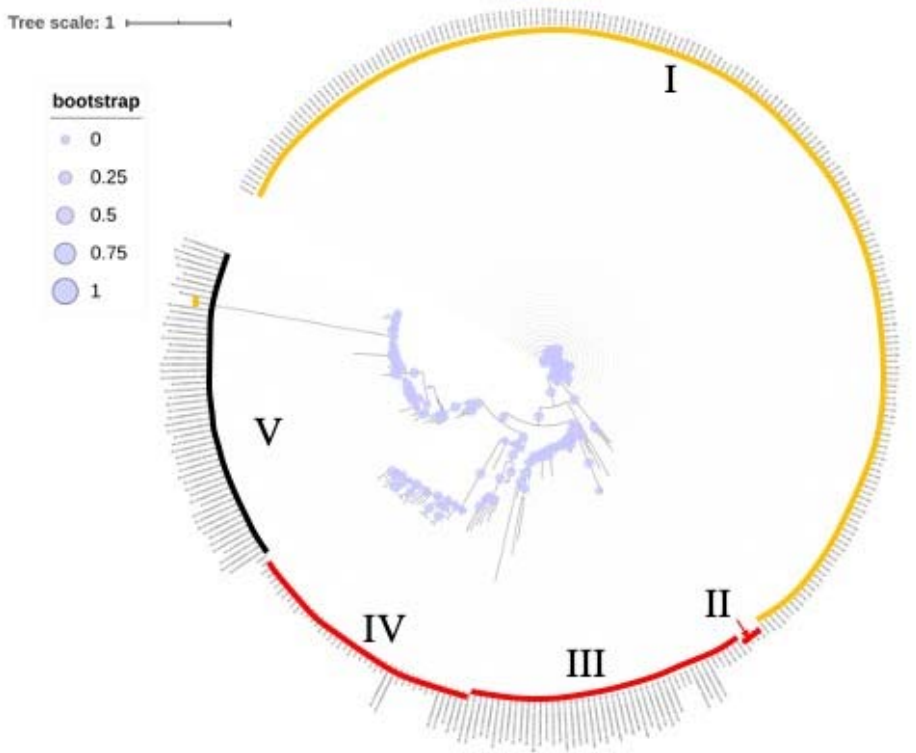

513

514

515 
516 Fig. 4 Distributions of long Am_MLEs. LG_1 to LG_16 indicate the chromosome names of $A$.

517 mellifera. Yellow markers indicate long Am_MLEs. Bold yellow markers indicate two MLEs

518 placed at very close positions to each other. The figure was drawn by chromoMap

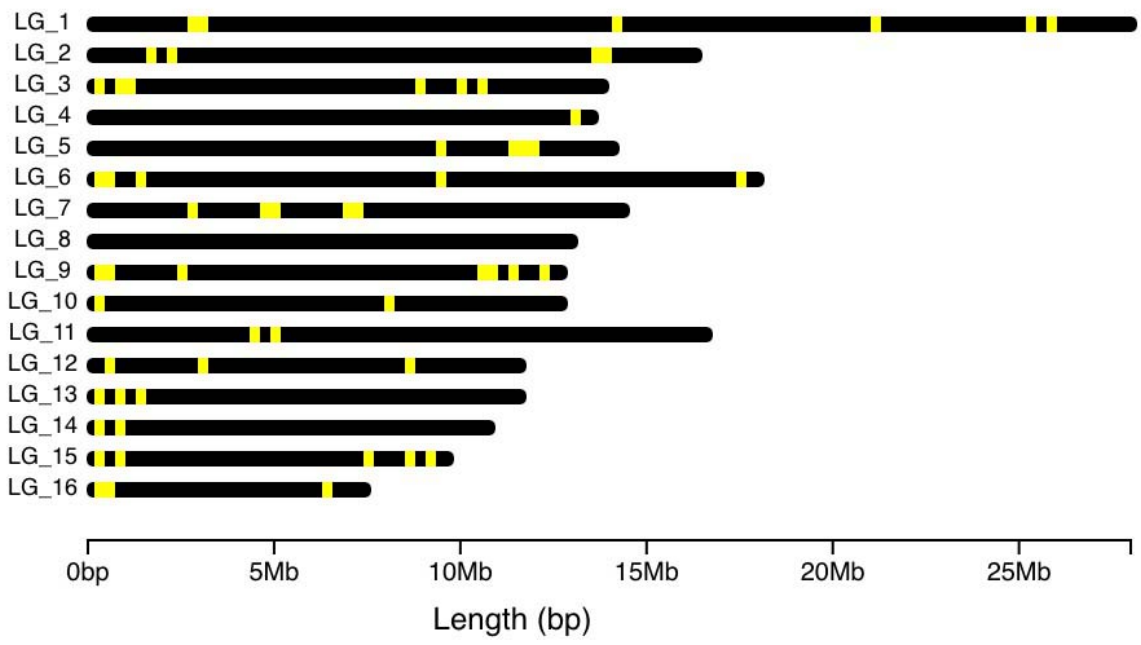

520 Fig. 5 Phylogenetic tree of transposases of Apis long MLEs

521 Phylogenetic tree of predicted transposases of Apis long MLEs. Orange- and red-curved lines in

522 these figures indicate clusters including only Am_MLEs and multiple Apis species MLEs.

523 These original figures downloaded from iTOL are shown in supporting data 13. 
bioRxiv preprint doi: https://doi.org/10.1101/2020.04.15.035063; this version posted November 30, 2020. The copyright holder for this preprint (which was not certified by peer review) is the author/funder, who has granted bioRxiv a license to display the preprint in perpetuity. It is made available under aCC-BY 4.0 International license.

524

525

526

527
Fig. 6 Evolutional insight of Apis MLEs

Tree scale: $1 \longmapsto$

bootstrap

0

0.25

0.5

0.75

( 1

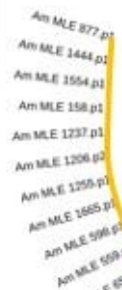

Schematic summary of evolutionary insights into Apis MLEs revealed by this study.

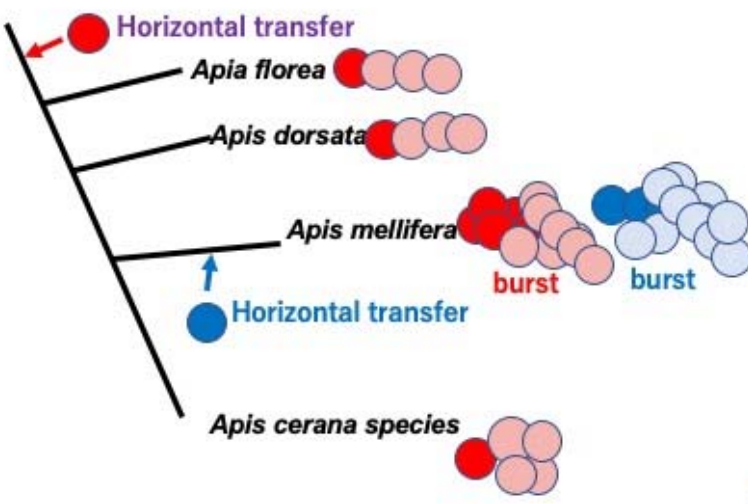

MLE which Apis cerana Mariner

transposase-like sequence

\section{MLE which lost mobility}

MLEs which carry Camar1

transposase-like sequence

MLE which lost mobility 
531 Table 1 Apis genome assemblies used in the present study

\begin{tabular}{|l|l|r|}
\hline Organism name & GenBank assembly accession & Contig N50 \\
\hline A. mellifera & GCA_003254395.2 & $5,382,476$ \\
\hline A. cerana japonica & GCA_002217905.1 & 179,487 \\
\hline A. cerana cerana Korea native & GCA_001442555.1 & 43,751 \\
\hline A. cerana cerana China native & GCA_002290385.1 & 21,160 \\
\hline A. dorsata & GCA_009792835.1 & 30,868 \\
\hline A. florea & GCA_000184785.2 & 24,915 \\
\hline A. mellifera carnica (Carniolan honeybee) & GCA_013841245.1 & $2,692,667$ \\
\hline A. mellifera intermissa & GCA_000819425.1 & 504 \\
\hline A. mellifera caucasica (Caucasian honeybee) & GCA_013841205.1 & $3,303,520$ \\
\hline A. mellifera mellifera (German honeybee) & GCA_003314205.1, & $5,131,172$ \\
\hline A. cerana cerana China native (new version) & GCA_011100585.1 & $3,898,192$ \\
\hline
\end{tabular}

532 Table 2 Numbers of long MLEs (1 kbp < length)

\begin{tabular}{|l|r|r|}
\hline Organism name & Number of long MLEs & Total number of MLEs \\
\hline A. mellifera & 80 & 2,145 \\
\hline A. cerana japonica & 7 & 158 \\
\hline A. cerana cerana Korea native & 6 & 140 \\
\hline A. cerana cerana China native & 7 & 145 \\
\hline A. dorsata & 3 & 163 \\
\hline
\end{tabular}


bioRxiv preprint doi: https://doi.org/10.1101/2020.04.15.035063; this version posted November 30, 2020. The copyright holder for this preprint (which was not certified by peer review) is the author/funder, who has granted bioRxiv a license to display the preprint in perpetuity. It is made available under aCC-BY 4.0 International license.

\begin{tabular}{|l|r|r|}
\hline A. florea & 0 & 163 \\
\hline
\end{tabular}

533 